\title{
Noun-modifier order in a semantic verification task
}

\author{
BARBARA TVERSKY, SIMHA HAVOUSHA, and ARIN POLLER \\ Hebrew University of Jerusalem
}

\begin{abstract}
In a semantic verification task, presenting the noun before its modifier yielded faster reaction times than did the opposite order of presentation. This finding held for two languages and for both concrete (food terms) and abstract (country names) terms and is thus not attributable to natural word order or to type of stimulus. The results can be explained in terms of spreading activation (Collins \& Loftus, 1975). A post hoc analysis revealed that concrete nouns are verified faster when paired with concrete rather than abstract modifiers, but that abstract nouns are verified faster when paired with abstract rather than concrete modifiers. An additional group of subjects, asked to produce attributes to nouns, produced more concrete than abstract modifiers to contrete nouns, but more abstract than concrete modifiers to abstract nouns. This result was taken as suggestive of possible differences in internal structure of concrete and abstract categories.
\end{abstract}

In semantic memory tasks, the time taken to verify propositions has been shown to reflect the distance or relatedness of the constituents of the propositions. Thus, subjects respond faster to "A robin is a bird" than to "A chicken is a bird" because "robin" is more related to "bird" than is "chicken," where relatedness is independently measured (Smith, Shoben, \& Rips, 1974). Recent research has suggested that verification occurs in a context or network, so that the time to verify a proposition is affected not only by the relatedness of its particular constituents, but also by the interrelatedness of the other concepts likely to be activated by the constituents (Collins \& Loftus, 1975). Consider an example of Collins and Loftus, a proposition like "A fire engine is red." The concept "fire engine" is likely to activate related concepts such as other vehicles and the elements of a fire scene; these concepts in turn would reactivate each other, leading to a dense, narrow pattern of activation. On the other hand, "red" would activate other red objects and other color names and objects possessing these colors, so that activation would spread shallowly across a broad area of the network. Put differently, the concepts activated by a noun or a superordinate noun category are likely to be richly interlinked, whereas the concepts activated by a modifier are not likely to be interlinked. This analysis leads quite naturally to predicting asymmetries in verification time; if the network is entered first at "fire engine" and then at "red," verification time should be faster than in the opposite case, because of the

This research was supported by a grant from the Human Development Institute of the Hebrew University of Jerusalem. Requests for reprints should be sent to Barbara Tversky, Department of Psychology, Stanford University, Stanford, California 94305. The authors are indebted to Fred Conrad for running Experiment 3. differing patterns of spread of activation. The present experiment tests this hypothesis using two domains of nouns, food and country terms, and attributes modifying them.

Empirical support for the hypothesis has been obtained for superordinate terms in a production task by Freedman and Loftus (1971). These experimenters found that subjects could produce a "fruit that is round" $.23 \mathrm{sec}$ faster than "a round fruit." We wanted to test the effect of order of presentation in a verification task because of a competing interference hypothesis that ascertaining any particular attribute might be slower when several other attributes are simultaneously aroused. Thus, reaction time (RT) to "a cherry is round" might be slower because of interference from other attributes aroused by "cherry" (e.g., red, fruit, sweet, small). The spread of activation from "round" presented first would be shallower and broader and less likely to produce interference. If such interference is a factor in verification, although not in production, it should be greater for concrete stimuli, that could be imaged with many of their properties, than for abstract stimuli, such as country terms, that are more difficult to image. Thus, the experiment included both sorts of stimuli.

A final consideration in designing the experiment was the natural word order in language. Since we had reason to believe that the verification task would produce results opposite those of the production task, we ran the experiment in Hebrew, where the modifier normally follows the noun, and later replicated in English, where modifiers normally precede nouns.

\section{EXPERIMENT 1}

\section{Method}

Subjects. Subjects were 32 right-handed native Hebrew speakers, students at the Hebrew University, who were paid or 
received course credit for their participation in the experiment.

Stimuli. The stimuli were 40 country-attribute pairs and 40 food-attribute pairs. There were 10 country attributes (large, small, coastal, inland, northern hemisphere-denoted "north," southern hemisphere, warm, cold, wealthy, and poor), with four different instances per attribute. There were 10 food attributes (red, green, round, long, large, small, hard, soft, fruit, and vegetable), with four different instances per attribute. Note that in each case, the 10 attributes were formed from five pairs of opposing terms. The countries and foods were pretested to make sure that they were familiar and that they were clear-cut examples of the modifiers attributed to them. The stimuli were typed (in Hebrew) on slides, separate slides for nouns and modifiers.

Design. Half the subjects responded "yes" with the right index finger and half with the left index finger. The major variables of interest, (attribute) before vs. after, food vs. country, and yes vs. no, were all within-subjects variables, counterbalanced as follows. Country and food names appeared in blocks; for half the subjects, countries were first and food second, and for the other subjects, food terms preceded country terms. For each subject, the modifier preceded the country or food name on half the trials, and followed the noun on the other half. Practice effects of position were counterbalanced such that a quarter of the subjects received each of the following orders: before, after, after, before; after, before, before, after; before, after, before, after; after, before, after, before. Each country and each food appeared only once for each subject; for half the subjects, half the nouns were correctly paired (e.g., small-Holland, green-pea) and the rest were incorrectly paired (e.g., Iceland-warm, olive-large), and the pairing was reversed for the other half of the subjects. The preceding four counterbalancing measures yielded 32 conditions, 1 for each subject.

Procedure. Subjects were run individually. At the start of each session, the entire experiment was explained to the subject, and he was familiarized with the attributes for both foods and countries, with examples of foods and countries not used in the actual experiment. He was given practice on the apparatus with practice trials using animal names as stimuli. Subjects were instructed to respond as quickly as possible without making errors.

The subject initiated each trial by pressing a foot lever. This caused the first slide to appear on the right side of the screen. Exactly $1 \mathrm{sec}$ after onset of the first slide, the second slide appeared to its left (Hebrew is read from right to left), and the timer, accurate to $1 \mathrm{msec}$, began. The subject responded by pressing the appropriate key, thereby stopping the timer and removing both slides from the screen. The experimenter's feedback was the cue to initiate the next trial.

Before each of the four trial blocks of 20 trials each (before vs. after by food vs. countries), the subject was informed of the nature of the stimuli of the following block. Sessions took about $30 \mathrm{~min}$.

\section{Results}

Error rates were all below $3 \%$, and erroneous responses were omitted from the analysis. An analysis of variance on the RT data yielded significant effects for food faster than countries $[F(1,31)=12.98, \mathrm{p}<.01$, $\mathrm{MSe}=.650]$, for "yes" faster than "no" $[\mathrm{F}(1,31)=8.79$, $\mathrm{p}<.01, \mathrm{MSe}=.147]$, and for attribute after faster than before $[F(1,31)=17.03, \mathrm{p}<.001, \mathrm{MSe}=.246]$. Mean RTs for these variables are reported in Table 1, along with the comparable data from Experiment 2. The only significant interaction was that between food vs. countries and yes vs. no $[\mathrm{F}(1,31)=5.33, \mathrm{p}<.05$, $\mathrm{MSe}=.173]$. The form of this interaction was that the "yes" responses were much faster than "no" responses for countries $(83 \mathrm{msec})$, but not much faster for food (7 msec).

Post hoc analyses were performed on RTs to various kinds of attributes. Attributes were called "concrete" if they referred to visible features of food or to visible features of maps of countries, and "abstract" otherwise. The concrete features of food thereby classified were color, shape, and size, and the abstract features were texture and category. For countries, the concrete features were size, coastal-inland, and hemisphere, and the abstract features were climate and finances. The noun terms can also be classed as concrete or abstract in terms of visibility. In this sense, food terms are concrete and country terms, abstract. The results of this analysis are reported in Table 2, along with the comparable data from Experiment 2. It is evident from Table 2 that for concrete stimuli (food), concrete attributes are more rapidly verified than abstract attributes $(t=4.33, p<.001)$, but for abstract stimuli (countries), abstract attributes are more quickly verified than concrete $(\mathrm{t}=4.40, \mathrm{p}<.001)$. RTs to concrete vs. abstract attributes do not differ overall; moreover, verification of abstract properties takes about the same amount of time for both food and country terms. Concrete attributes contribute the largest source of variance; for food terms, they are very rapidly verified and for countries, they are very slowly verified.

Post hoc analyses were also performed on attribute pairs that seemed to have a marked and an unmarked member (e.g., large-small) to determine if markedness (Clark \& Chase, 1972) affected RT; the results of these analyses were negative.

\section{EXPERIMENT 2}

In the natural word order of Hebrew, modifiers follow the nouns they modify. In order to determine

Table 1

Mean Reaction Times in Seconds From Experiments 1 and 2

\begin{tabular}{|c|c|c|c|c|c|c|c|c|}
\hline & \multicolumn{4}{|c|}{ Attribute Before } & \multicolumn{4}{|c|}{ Attribute After } \\
\hline & \multicolumn{2}{|c|}{ Food } & \multicolumn{2}{|c|}{ Countries } & \multicolumn{2}{|c|}{ Food } & \multicolumn{2}{|c|}{ Countries } \\
\hline & Hebrew & English & Hebrew & English & Hebrew & English & Hebrew & English \\
\hline Yes & 1.405 & 1.301 & 1.500 & 1.463 & 1.321 & 1.180 & 1.380 & 1.374 \\
\hline No & 1.393 & 1.333 & 1.560 & 1.484 & 1.347 & 1.230 & 1.486 & 1.510 \\
\hline
\end{tabular}


Table 2

Mean Reaction Times for Food and Country Terms by Attribute Type and Language

\begin{tabular}{lccccccc}
\hline & \multicolumn{3}{c}{ Hebrew } & & \multicolumn{3}{c}{ English } \\
\cline { 2 - 4 } \cline { 6 - 8 } & Food & Countries & Mean & & Food & Countries & Mean \\
\hline Concrete & 1.327 & 1.525 & 1.426 & & 1.219 & 1.501 & 1.360 \\
Abstract & 1.426 & 1.416 & 1.421 & & 1.324 & 1.393 & 1.359 \\
\hline
\end{tabular}

whether the results of the previous experiment were due to natural word order or some property of semantic memory, the experiment was replicated in English, where the natural word order is reversed.

\section{Method}

Subjects. Subjects were 16 right-handed native English speakers, students at the Hebrew University, who were paid or received course credit for their participation in the experiment.

Stimuli. The stimuli of the previous experiment were translated into English and typed on slides. Six food terms were changed to foods more familiar to English speakers.

Design. The design of Experiment 1 was cut in half, to accommodate the reduced number of subjects, by using only four of the eight possible conditions of order of terms (foodcountry) by order of before-after.

Procedure. The procedure was identical to Experiment 1, except that the first stimulus appeared on the right side of the screen, and the second on the left, to correspond to reading in English.

\section{Results}

Error rates were all below 3\%, and erroneous responses were eliminated from the analyses. An analysis of variance on the data revealed that food terms were responded to significantly faster than countries $[\mathrm{F}(1,15)=11.51, \mathrm{p}<.01, \mathrm{MSe}=1.079]$, and modifier after was significantly faster than modifier before $[\mathrm{F}(1,15)=8.34, \mathrm{p}<.05, \mathrm{MSe}=.195]$. "Yes" responses were faster than "no" responses, but this difference fell short of significance $[F(1,15)=3.25]$. None of the interactions was significant. Mean RTs are reported in Table 1.

The same post hoc analysis on attribute types was performed, with similar results, appearing in Table 2. For concrete stimuli (foods), concrete attributes were verified faster than abstract $(\mathrm{t}=3.55, \mathrm{p}<.001)$, and the reverse held for abstract (countries) stimuli $(t=3.09$, $\mathrm{p}<.01)$. The same pattern of results appeared in English as in Hebrew: no overall difference between concrete and abstract verification times, no difference to verify abstract attributes between food and country terms, and a large difference between food and country terms on concrete attributes.

As in Experiment 1, no effects of attribute markedness were found in RT.

\section{EXPERIMENT 3}

In both Experiments 1 and 2, we found an effect of matching concreteness or abstractness of nouns and modifiers. Subjects responded faster to concrete nouns when paired with concrete modifiers and to abstract nouns paired with abstract modifiers than to concreteabstract or abstract-concrete pairs. In order to ascertain whether this effect was due to higher availability of concrete modifiers to concrete nouns and abstract modifiers to abstract nouns, we asked another group of subjects to provide modifiers (attributes) to the nouns presented in Experiment 2.

\section{Method}

Subjects. Subjects were 16 Stanford students participating in a memory experiment, who were paid or received course credit for their participation.

Stimuli. The stimuli were the 40 names of foods and 40 names of countries used in Experiment 2. Each subject received five of each, grouped by foods or countries, but randomly ordered within a category. The entire list appeared in one order across the first eight subjects and in the opposite order across the second eight subjects.

Procedure. Subjects were asked to write "the first, few salient attributes that come to mind for each country and food," and they were given $3 \mathrm{~min}$ for the food terms and $3 \mathrm{~min}$ for the country terms.

\section{Results}

Food terms elicited more attributes (4.31 average per subject per item) than country terms (3.54 average), a significant finding $[\mathrm{t}(30)=1.84, \mathrm{p}<.05] ; 12$ out of the 16 subjects gave more attributes to foods than to countries. Of the attributes elicited by food terms, 38\% were of the type listed in Experiments 1 and 2 (color, size, shape, hardness, and category), but of the attributes elicited by countries, only $13 \%$ were of the type appearing in Experiments 1 and 2 (size, coastal-inland, hemisphere, climate, and wealth). This finding is also significant $[\mathrm{t}(30)=5.85, \mathrm{p}<.001]$ and was true for all subjects. A two-way analysis of variance was performed on those attributes elicited by the present subjects, specified as concrete or abstract in Experiments 1 and 2 for food and country terms. The mean numbers of attributes per subject per item are reported in Table 3 , by type of term and type of attribute. More specified attributes were elicited by food terms than by country terms $[\mathrm{F}(1,30)=45.82, \mathrm{p}<.001$, $\mathrm{MSe}=.11]$, and more concrete attributes were elicited than abstract attributes $[\mathrm{F}(1,30)=29.16, \mathrm{p}<.001$, MSe $=.11]$. The expected interaction was also significant $[\mathrm{F}(1,30)=47.90, \mathrm{p}<.001, \mathrm{MSe}=.11]$, of the

\section{Table 3}

Mean Number of Specified Concrete and Abstract Attributes Produced per Subject per Food or Country Term

\begin{tabular}{lccc}
\hline & \multicolumn{3}{c}{ Attributes } \\
\cline { 2 - 4 } Terms & Concrete & Abstract & Sum \\
\hline Food (Concrete) & 1.29 & .28 & 1.57 \\
Country (Abstract) & .16 & .29 & .45 \\
Sum & 1.47 & .57 & \\
\hline
\end{tabular}


form that food terms elicit more specified concrete attributes than abstract attributes, whereas country terms elicit more specified abstract attributes than concrete attributes. Thus, for the subset of attributes specified in Experiments 1 and 2, the frequency of type of attribute elicited (concrete or abstract) interacts with the type of term (concrete or abstract) in exactly the same pattern as the RTs in Experiments 1 and 2.

\section{DISCUSSION}

The major findings of Experiment 1 were replicated in Experiment 2. When the food or country term preceded its modifier, verification times were faster than when the modifier preceded the noun. Interestingly, the size of this effect was about the same in Hebrew $(81 \mathrm{msec})$, where it corresponded to natural word order, and in English $(71 \mathrm{msec})$, where it opposed natural word order. Correct rejections were, on the whole, slower than correct acceptances, an effect typically obtained in RT studies using semantic or perceptual stimuli.

The largest effects, in terms of RT, are attributable to the nature of the stimuli. Food terms were responded to faster than countries (by $114 \mathrm{msec}$ in Hebrew, $197 \mathrm{msec}$ in English). Food terms are typically more frequent in language than are country names; they are also more concrete and more vivid and acquired at an earlier age. Since these properties of words are correlated, there is no way of knowing which accounts for shorter RTs in these experiments. The second aspect of the stimuli reflected in RT was the interaction between concreteness of nouns and of modifiers. When the noun and modifier are matched on concreteness or abstractness, verification times are more than $100 \mathrm{msec}$ faster than when a concrete noun is paired with an abstract modifier or an abstract noun is paired with a concrete modifier. This effect appears in both languages. The same pattern of findings emerged when subjects were asked to list attributes describing country and food terms. The abstract terms (country names) elicited more abstract than concrete attributes, while the concrete terms (food names) elicited more concrete than abstract modifiers. These findings, of faster RTs to concrete-concrete and abstract-abstract pairs and of greater availability of concrete modifiers to concrete nouns and of abstract modifiers to abstract nouns, can only be regarded as suggestive. The experiment was not designed to find such differences, only one domain of concrete and one domain of abstract were investigated, and the operational definitions of concrete and abstract were post hoc and not without problems. Nevertheless, the results are intriguing in that they suggest that the internal structure of categories (Rosch, 1975) that are not pictureable may differ from those that are concrete.

The effect of word order on verification time extends the Freedman and Loftus (1971) findings for production to a verification task and another language. The spreading-activation theory of Collins and Loftus (1975) accounts for both results in terms of differing patterns of activation aroused by noun concepts as opposed to modifiers. In fact, weaker theories of semantic memory could adequately account for the findings. One only needs to assume asymmetry in the strength of relation between noun (superordinate or instance) and modifier, such that modifiers are more closely related to nouns than vice versa. Any adequate account of the findings must attribute some sort of primacy to nouns and noun categories, with an ancillary cross-index based on modifiers. Support for the primary status of nouns over modifiers also comes from studies of episodic memory (Lockhart, 1969). While activation in a semantic network is not essential to explain the present findings, it does seem useful in accounting for other phenomena, such as certain repetition effects (Collins \& Loftus, 1975). In any event, the present findings limit attempts to account for semantic memory to models that can account for asymmetric relations between nouns and modifiers.

\section{REFERENCES}

Collins, A., \& Loftus, E. F. A spreading-activation theory of semantic processing. Psychological Review, 1975, 82, 407-428.

Clark, H. H., \& Chase, W. G. On the process of comparing sentences against pictures. Cognitive Psychology, 1972, 3, 472-517.

Freedman, J. L., \& Loftus, E. F. Retrieval of words from longterm memory. Journal of Verbal Learning and Verbal Behavior, 1971, 10, 107-115.

LOCKHART, R. S. Retrieval assymetry in the recall of adjectives and nouns. Journal of Experimental Psychology, 1969, 79, 12-17.

Rosch, E. Cognitive representations of semantic categories. Journal of Experimental Psychology: General, 1975, 104, 192-233.

Smith, E. E., Shoben, E. J., \& Rips, L. J. Structure and process in semantic memory: A featural model for semantic decisions. Psychological Review, 1974, 81, 214-241.

(Received for publication September 28, 1978.) 